\title{
COMPETITIVE INTELLIGENCE: DE LOS ESTADOS A LAS EMPRESAS
}

\author{
Carlos Sánchez Hernández \\ Universidad Complutense de Madrid
}

Resumen.- La "Competitive Intelligence" es un área de conocimiento tradicional, aunque sea con otras denominaciones, entre las políticas exteriores, políticas militares e incluso servicios de inteligencia de muchos Estados. Pero en las últimas tres décadas, y de forma cada vez más creciente, ha destacado por trasladarse, con muchos de los métodos propios de los Estados, al mundo empresarial y de los negocios, de las multinacionales fundamentalmente, y de la empresa en general. Se trata de una disciplina relacionada con el conocimiento, el análisis, el desarrollo y el procesamiento de los datos con la finalidad de obtener un beneficio de tipo competitivo, conocer el entorno de la competencia empresarial e influír en el proceso de toma de decisiones, todo ello aplicado al campo de la libre competencia empresarial.

Palabras clave.- Competitive intelligence, competencia, marketing, análisis, empresa, beneficio.

\begin{abstract}
The "Competitive Intelligence" is an area of traditional knowledge, even with other denominations, including foreign policy, military policy and even the intelligence services of many states. But in the last three decades, and an increasingly growing, stressed by moving, with many of the States' own methods, the business world and business, mainly multinationals, and business in general. It is a related discipline with knowledge, analysis, development and data processing in order to gain competitive type, knowing the competitive business environment and influence the decision making process, all this applied to the field of business competition.
\end{abstract}

Keywords.- Competitive intelligence, competition, marketing, analysis, company, benefit.

\section{Introducción}

Frontera entre la República Federal Alemana y la República Democrática Alemana, 1983: las tropas estadounidenses y soviéticas se observan entre sí, como desde hace treinta años, tratando de desenmascarar las intenciones del adversario. A comienzos de 1983, en unas maniobras militares del Pacto de Varsovia, los estadounidenses observan, en los movimientos de tropas soviéticas, cómo éstas movilizan 200 carros de combate, 20 lanzaderas móviles de misiles, y unos 20.000 soldados. Inmediatamente los observadores estadounidenses de la frontera inter-alemana redactan un informe en el que, en base a esos datos, elaboran una hipótesis basada en el procesamiento lógico de dichos datos, llegando a la conclusión de que se trata de unas maniobras militares de carácter ofensivo, destinadas a impresionar e incluso provocar a las tropas de la OTAN con una finalidad estratégica, tratando de alterar el frágil equilibrio de poder en Centroeuropa, acercando misiles ICBM (misiles 
intercontinentales) a la frontera, osea, acercándolos unos cien kilómetros más al territorio de los aliados de EE.UU, y simbólicamente al propio territorio norteamericano, que sería el destinatario final de ésta clase de misiles nucleares. El informe de los analistas militares sobre el terreno es enviado al Pentágono, donde es perfilado dándole retoques militares pero sobre todo una lectura política destinada al Secretario de Defensa, Caspar Weinberger: los soviéticos pretenden dar una vuelta de tuerca en su juego nuclear en Centroeuropa para obligar a reaccionar a la OTAN y medir dicha reacción, pretendiendo, si la respuesta estadounidense no es firme, escalar en la tensión y apuntarse una victoria estratégica. El Pentágono y el Secretario de Defensa recomendarán, en base a éste informe, al presidente Ronald Reagan, al gabinete presidencial, y al Consejo de Seguridad Nacional trazar una respuesta política y militar contundente para enviar un mensaje claro a Moscú, en el sentido de que la exhibición de fuerza no resultaría; se movilizarán fuerzas militares de la OTAN en la frontera inter-alemana en un número superior incluso al de la movilización soviética, se romperán e interrumpirán indefinidamente las conversaciones sobre desarme, y se determinará un "Defcom 3" en las fuerzas armadas de EE.UU, un paso cercano a la guerra.

Ésta situación hipotética, propia del último año tenso de la Guerra Fría, ilustra lo que era y es la inteligencia en el mundo político-militar, osea en el ámbito estatal. Esto puede trasladarse a la empresa, y de hecho se está haciendo, por medio de la Competitive Intelligence, buscándose lógicamente no unos fines violentos propios del juego militar, pero sí empresariales. Pongamos un ejemplo: dos compañías de seguros, las dos gigantes aseguradoras del mundo, se observan: una de ellas (cía. A) elabora un informe en base a los datos que la otra (cía. B) deja entrever en sus informes corporativos públicos, los que "cuelga" en su web para que sus clientes puedan comprobar su solvencia; balances, beneficios, cuentas de explotación, apuestas por sectores, apuestas por regiones del mundo, nuevas inversiones, absorciones previstas y ventas, despidos o incorporaciones de personal. Un analista de la compañía A elabora un informe: la compañía B ganó en el ejercicio fiscal pasado un $32 \%$ más que en el anterior,y ello en el contexto de una grave crisis financiera que asola al mundo desde Agosto de 2007. Apostó por invertir, según se observa en sus informes públicos, en el emergente mercado asiático, y pretende continuar con esa tendencia al observar cómo ha incorporado a cuarenta altos ejecutivos y a veinticinco de ellos los ha destinado a sus instalaciones de Asia. Sus intenciones son, según se aprecia en sus análisis publicados en su web, seguir ampliando su presencia en Asia donde está obteniendo una buenísima parte de sus beneficios totales. Por último, apuesta tanto por el sector asegurador del ramo vida-riesgo, como por invertir en productos financieros derivados para maximizar sus beneficios. La cía. A puede sacar ventaja de toda ésta información de su principal competencia tras analizarla y procesarla, para imitarla y seguir su tendencia si realmente se revela como exitosa, o bien para contrarrestar el éxito de su oponente.

Esto es en esencia, y en un breve ejemplo aplicado al ámbito empresarial, la Competitive Intelligent. 


\section{2. ¿Qué es la Competitive Intelligence? El Marco de la Business Intelligence}

El concepto de "Competitive Intelligence" (Inteligencia Competitiva) se engloba dentro del más amplio concepto de "Business Intelligence" (Inteligencia Empresarial). La Business Intelligence es el término más amplio que existe en todo lo relativo al empleo de la inteligencia en las organizaciones empresariales. Se trata de toda una disciplina que ha ido surgiendo a partir de diversas disciplinas del conocimiento empresarial, dando lugar a una variedad de áreas del conocimiento englobadas todas ellas dentro de la propia Business Intelligence y de la Market Intelligence (Inteligencia de Mercados).

La Business Intelligence, como forma genérica de referirse a todo lo relacionado con el uso de la inteligencia en el ámbito de la empresa, está siendo ampliamente usada en el ámbito de la tecnología con diversos significados en forma de apartados, como es el caso de la "Strategic Foresight" (Inteligencia Estratégica), la "Corporate Intelligence" (Inteligencia Corporativa), y otras más específicas ceñidas a otras áreas del conocimiento.

Se conoce como "Business Intelligence" al conjunto de estrategias y de herramientas orientadas a la gestión de conocimiento mediante el análisis de datos existentes en una organización empresarial. El análisis y procesamiento de la información debe diferenciar entre los distintos tipos de información que existen; así, se distinguen entre "datos", que es algo vago y genérico (100 millones de $€$ ), información a secas, que es algo más preciso (la facturación de la cía. A en 2010 fue de 100 millones de $€$ ), y el conocimiento, un tipo de información obtenida mediante el análisis y procesamiento profundos de la información. Es entonces cuando entra en funcionamiento la Business Intelligent ya que una vez analizada toda la información y obtenido el conocimiento en forma de conclusiones aleccionadoras, se pueden elaborar conductas empresariales consecuentes, o estrategias empresariales. Al haber logrado la información de todas las partes posibles de una empresa, ya sea la propia ú otra, se ha obtenido el conocimiento y se ha establecido la conducta a seguir, señalando sus debilidades, amenazas, fortalezas y oportunidades (DAFO).

La finalidad principal de la Business Intelligent es facilitar el proceso de toma de decisiones en una empresa. Engloba el análisis y comprensión del funcionamiento de una empresa en un momento dado, para a partir de ahí anticiparse a acontecimientos futuros y ofrecer conocimientos para confirmar, rechazar o corregir las decisiones empresariales. Las herramientas de inteligencia se basan en el uso de un sistema de información de inteligencia elaborado a partir de una variedad de datos conseguidos a su vez de los datos de producción, a base de información relacionada con la empresa o sus ámbitos, y con datos económico-financieros.

Una técnica ampliamente utilizada para obtener los datos es la denominada "extraer, transformar y cargar", que permite extraer los datos de distintas 
fuentes, filtrarlos y homogeneizarlos para finalmente almacenarlos en un "almacén de datos". Ese almacén será renovado según su comportamiento y utilidad, según el nivel de éxito que aporte a la empresa que lo usa. Incluso esa información puede llegar a ser compartida y comercializada a otras empresas, como un bien más. Finalmente, las herramientas de inteligencia de análisis hacen posible la manipulación y transformación, llegándose a crear un "cuadro de mando integral" que sirve como materia prima para la elaboración de informes. Éstas herramientas y métodos tienen características propias, la primera de ellas la accesibilidad a la información, ya que los datos son la fuente principal, ya que éstas herramientas deben garantizar el acceso de los usuarios a los datos. Otra característica importante que deben tener es el apoyo en la toma de decisiones, ya que se pretende avanzar en la presentación de la información, de forma que los usuarios tengan acceso a herramientas analíticas que les permitan emplear sólo aquellos datos que les sean útiles. Por último, las herramientas deben tener una clara orientación al usuario final, de forma que éste pueda usarlas con cierta comodidad.

De forma analítica, y según su nivel de complejidad, se pueden clasificar las soluciones por niveles dentro de la Business Intelligent en:

- Querys and Reports (consultas e informes)

- OLAP's, "on-line analytic processing

- Data Mining ("ingeniería” de datos)

- Diversos sistemas de estudio empresarial basados en series temporales

\section{La Vigilancia: el Antecedente Histórico de la Competitive Intelligent}

Siempre se ha considerado que "vigilar a la competencia" era un método inestimable para elaborar correctamente un buen proceso de toma de decisiones. Desde hace décadas, el denominado "espionaje industrial" ha sido un instrumento fundamental para el logro de estrategias empresariales. Ya en el Siglo XVIII en Suecia, un país pionero en actividades de inteligencia empresarial, existía una revista llamada "Den Goteborg Spionen" que se dedicaba a suministrar información sobre las novedades en todo lo relativo a las tecnologías desarrolladas en el extranjero, para que las pudieran incorporar las empresas suecas. Y es que a día de hoy, una empresa que quiera ser competitiva está obligada a mantenerse perfectamente informada sobre el ambiente empresarial de su sector con el propósito de identificar todos los puntos que supongan un motivo de amenaza o de beneficio. Es así como surgen los conceptos de vigilancia e inteligencia competitiva, diferenciados del espionaje industrial porque no violan los límites marcados por la ética y por la ley.

Una definición comúnmente aceptada de "sistema de vigilancia tecnológica" sería ésta: "es la búsqueda, detección, análisis y comunicación a los directivos de la empresa de informaciones orientadas a la toma de decisiones sobre amenazas y oportunidades externas en el ámbito de la ciencia y la tecnología" 
(definición del profesor Escorsa). Otra definición (profesor Morcillo) sería "un sistema de vigilancia tecnológica consiste en analizar el comportamiento innovador de los competidores directos e indirectos, explorar todas las fuentes de información (libros, bases de datos, patentes, etc ...), examinar los productos existentes en el mercado (tecnología incorporada) y asistir a ferias y congresos para posicionarse respecto a los demás competidores y tomar así conocimiento de las competencias tecnológicas que predominarán en un futuro más o menos próximo. Todo ello sin perder de vista la capacidad tecnológica presente y la que estará en condiciones de desarrollar la empresa para enfrentarse a nuevos retos". De forma consensuada y sintética, la vigilancia tecnológica es la forma organizada, selectiva y permanente de captar información del exterior, analizarla y convertirla en conocimiento para poder anticiparse a los cambios y procurar la toma de decisiones con menor riesgo.

Otro concepto ligado a la vigilancia tecnológica es la Innovación Tecnológica, que es el conjunto de acciones simultáneas de búsqueda, análisis y explotación de la información obtenida legalmente, acciones útiles para desarrollar las estrategias de una organización. Todo esto incluye la introducción de productos y procesos nuevos partiendo de la creación de conocimiento sobre los medios empleados, separándose según el objeto en innovación de procesos (instalación de nuevos procesos de producción que redundarán en una mejor productividad, mejor racionalización del proceso industrial, y mejora en los costes), o innovación de productos (fabricación y comercialización de nuevos productos, denominada innovación radical, o productos ya existentes mejorados, denominada innovación gradual).

Con la evolución del proceso industrial, en la época actual, sobre todo a partir de la II ${ }^{a}$ Guerra Mundial, y más aún con la "Revolución capitalista" acaecida en la década de los 1980's consistente en que para finales de esa década el capitalismo se convirtió en el único sistema económico posible en casi todo el planeta, y la subsiguiente Globalización iniciada en los 1990's, proliferaron todos éstos términos relativos a la competencia industrial. Efectivamente, fue a partir de los 1980's cuando la Vigilancia Tecnológica y la Inteligencia Competitiva nacen en el seno de la cultura empresarial, sobre todo en las grandes multinacionales, más dispuestas y con más posibilidades para ejercer éstas funciones. 1986 fue un año clave, ya que en Estados Unidos se constituye la Society of Competitive Intelligence Professionals (SCIP), que en la actualidad es la asociación sobre Competitive Intelligent más importante del mundo y que tiene ramificaciones y presencia en Europa y Japón, contando ya con 6.000 socios, algunos de ellos ejecutivos y empleados de empresas presentes en el ranking "Fortune 500". En España la única asociación existente es la ASEPIC (Asociación Española para la Promoción de la Inteligencia Competitiva).

Si Estados Unidos fue el pionero en todo lo relativo a Vigilancia Tecnológica, un país especialmente avanzado es Japón, de quien se estima que ésta disciplina es responsable de hasta un sorprendente $1,5 \%$ de sus exportaciones. En Europa sin embargo aún se asiste a un cierto retraso, siendo Alemania y Gran Bretaña los países más introducidos en lo relativo a Competitive Intelligent. 
España más en concreto se sitúa aún bastante retrasada, si bien importantes de sus empresas de los sectores farmacéutico y químico muestran progresos. Académicamente hablando, destacan en España las actividades del Consejo Superior de Investigaciones Científicas (CSIC), especialista en técnicas bibliométricas. También destacan la Universidad Carlos III (Madrid), el Instituto Superior de Marketing (Madrid), en Cataluña la Universidad Pompeu-Fabra, la Universidad Oberta de Cataluña y el Instituto Catalán de Tecnología, y en Valencia la Universidad Politécnica. Cabe destacar, en una iniciativa novedosa por tratar de unir el mundo de las instituciones propias del Aparato del Estado con el ámbito académico y empresarial, el nuevo Máster Interuniversitario en Analista de Inteligencia que se inaugura éste curso académico 2010-11, impulsado combinadamente por el Centro Nacional de Inteligencia (CNI), el Ministerio de Defensa, y las universidades madrileñas Rey Juan Carlos y Carlos III. Por último, el gobierno español ha creado un Observatorio Tecnológico, una colaboración para los docentes basado en la observación y evolución de la tecnología informática para analizarla y estudiarla, aplicándola en los niveles educativos no-universitarios.

En el mundo, y en España, cada vez proliferan más las empresas consultoras en materia de vigilancia, dándose cuenta de la importancia que tiene la competencia en el mundo empresarial. En 1999, Cartier pronunció la siguiente y posiblemente profética frase: "probablemente la vigilancia se convertirá en una actividad tan importante para la empresa como lo son hoy el marketing o la I+D”.

\section{Proceso y Etapas de Vigilancia Tecnológica e Inteligencia Competitiva}

Como ya hemos visto, de forma sintética, la Competitive Intelligent consiste en una serie de acciones que siguen una serie temporal consistente en la búsqueda, procesamiento (mediante el filtrado, clasificación y análisis), distribución, comprensión, explotación y finalmente protección de la información obtenida (siempre legalmente, acudiendo a fuentes "visibles" y públicas, sin recurrir al espionaje), información útil para los actores económicos de una organización, y útiles para que esos actores desarrollen sus estrategias individuales y colectivas.

Así, el esquema temporal de las etapas del proceso de vigilancia e inteligencia, gráficamente descrito, de la Competitive Intelligence, es éste:

$$
\begin{aligned}
& \text { 1-Identificar - } 2 \text {-Obtener -3 -Analizar -4 -Difundir } \\
& \text { 5-Utilizar -6 -Proteger }
\end{aligned}
$$

La Competitive Intelligence se convierte así en un proceso que "retroalimenta" en forma de feedback constantemente la planificación estratégica de una organización, en éste caso económica. Su principal objetivo es detectar señales de cambio, identificar y analizar tendencias, posibles cambios de rumbo y estrategias de la competencia, posibles amenazas nuevas, posibles 
nuevas oportunidades surgidas por el abandono de una estrategia de la competencia, etc ... El teatro competitivo siempre estará de éste modo actualizado.

La Competitive Intelligence abarca y absorbe a otra vertiente de la estrategia industrial: la Vigilancia Tecnológica, sumando a ésta la dimensión económica y empresarial (se trata en sus inicios de un concepto militar, recientemente adaptado al mundo de la empresa). Es por esto que se puede hablar abiertamente del sistema combinado "Inteligencia Competitiva-Vigilancia Tecnológica". La implementación de sistemas de Inteligencia CompetitivaVigilancia Tecnológica es clave hoy en día, para cualquier sector de actividad, si se quiere tener a la competencia "controlada".

En otro nivel, y centrándonos más en la Vigilancia Tecnológica, se puede hablar de "las secuencias de la vigilancia", describiéndolo tanto desde su partida como hasta su etapa final ó punto de llegada:

PLANIFICACIÓN Y DIREC. -Obtención de Información -Procesamiento Análisis -Difusión Resultados -Acciones -PLANIFICACIÓN Y DIREC.

Con éstas secuencias se logran los objetivos perseguidos que no son otros que la observación y el análisis del entorno científico, tecnológico y económico tanto actuales como venideros, para poder identificar las amenazas y oportunidades por medio de la difusión de las informaciones seleccionadas y analizadas, todo ello de gran utilidad para el proceso de toma de decisiones estratégicas. Los escenarios principales donde aplicar la Vigilancia son básicamente cuatro:

- la competencia

- la Vigilancia comercial (clientes, proveedores, mercado en general)

- la Vigilancia tecnológica (tecnologías disponibles)

- la Vigilancia del entorno (normativas, entorno económico, político)

Una Vigilancia más tradicional consiste en "asomarse" a realidades habituales de los mercados; las ferias de muestras sectoriales son una excelente forma. Otra es observar y analizar los productos de los competidores, así como cuestionar a los clientes y proveedores. Por último están las revistas especializadas. Una Vigilancia más avanzada consistiría en acudir a bases de datos, archivos técnicos, patentes, Internet, y a la denominada "minería de datos", una búsqueda profunda de nuevos datos. Algunas preguntas que todo aquel que practica una Vigilancia Tecnológica debe plantearse son: ¿cómo actúan mis competidores? ¿Quiénes lideran el mercado y el sondeo de mercado? ¿cuáles deben ser las principales líneas de investigación? ¿cuáles son las líneas de investigación emergentes?

A la larga, e incluso a medio plazo, el término "Inteligencia" tiende a sustituír al de "Vigilancia", ya que se le atribuye un carácter más activo. Además incluye una información mucho más analizada y tamizada, y más adaptada al proceso de toma de decisiones. "Inteligencia" es un término más amplio ya que integra a los propios resultados de la Vigilancia en distintos ámbitos (tecnológico, 
financiero, competitivo). La Vigilancia se centra en la detección, mientras que la Inteligencia tiene una visión más amplia, centrada en el posicionamiento estratégico de la empresa en su entorno. La Inteligencia no es sólo observación, sino una disciplina a la que se le puede atribuir una vertiente "ofensiva y defensiva" de la información.

Por último, cabe distinguir entre los conceptos de "Gestión del Conocimiento" e "Inteligencia Competitiva":

\section{Gestión del Conocimiento}

Dentro de la empresa

Se debe al pasado

Comparte conocimientos

\section{Inteligencia Competitiva}

En el exterior de la empresa

Se anticipa, se orienta al futuro

Detecta oportunidades

\section{5. ¿Es realmente efectiva la Competitive Intelligent?}

La auténtica aplicación efectiva de la Competitive Intelligent reside en conocer el entorno en constante cambio donde opera una empresa, lo cual puede reportar grandes ventajas estratégicas incluso a corto plazo.

Algunos estudios han revelado que cuando las empresas no son capaces de enterarse a tiempo del lanzamiento de productos o la bajada de precios por parte de los competidores, se resienten en el ejercicio en curso, al sentirse en inferioridad de condiciones respecto al competidor que maniobró antes que ella, sin su conocimiento, anticipándose y dejando a la empresa en cuestión fuera de mercado. Y es que la capacidad de respuesta frente a una ofensiva de precios o una nueva línea de productos de una empresa competidora es clave en el sector empresarial.

La Competitive Intelligent, por medio de sus métodos, ya suficientemente expuestos en este trabajo, pretende orientar la estrategia de la organización en el sentido de ajustarla mejor a su entorno, llegando a influír sobre éste para hacerlo más favorable. Sintéticamente, la Competitive Intelligent tiene dos grandes aplicaciones:

- disminución de la incertidumbre al disponer de más información sobre el entorno y los efectos que pueden tener los cambios de éste sobre el éxito de la empresa

- logro de una mayor probabilidad de éxito en la instauración de la estrategia, ya que la Competitive Intelligent proporciona formas de análisis para escrutar la reacción de la competencia ante un cambio de estrategia de la empresa 


\section{Ampliando Conceptos: Economía de la Información, Ventaja Competitiva y Otros Conceptos}

Las varias definiciones sobre Competitive Intelligent aportadas en éste trabajo quedarían incompletas si no incluímos la definición propia de la institución más importante a nivel mundial en cuanto a Competitive Intelligent, la SCIP norteamericana (Society of Competitive Intelligent Profesionals): "es un proceso ético y sistemático de recolección de información, análisis y diseminación pertinente, precisa, específica, oportuna, predecible y activa, acerca del ambiente de negocios, de los competidores y de la propia organización".

La Competitive Intelligent es el resultado de la combinación de varias subdisciplinas dentro de la ciencia económica, y los conceptos y términos de los que se ha nutrido han sido diversos. Su conceptualización teórica es aún, y a pesar de sus veinticinco años de existencia (nació como disciplina propia en Estados Unidos en 1986) escasa, y a pesar de que sus aplicaciones son amplias y su uso está ya extendido por todo el mundo.

El primer gran término conceptual y disciplinario indispensable para entender la Competitive Intelligent es la "Economía de la Información". Previo a éste concepto, está la consideración, generalmente aceptada hasta hace dos décadas, del denominado "equilibrio competitivo", consistente en la idea de que el grado de información que poseían todos los actores del mercado era igual para todos, y estaba al alcance de todos sin cortapisas; se trata de algo que en la actual Sociedad de la Información, desde que se extendió Internet y sus "autopistas de la información" a partir de 1995, está claramente en entredicho. Se trataba de una consideración basada en una situación de información perfecta, hoy día irreal. Por supuesto, se acepta que la importancia de la información en el mundo de los negocios es alta, y en éste segundo momento, que la información es imperfecta y "asimétrica". Y es que las asimetrías de información se producen en diversas situaciones, como cuando los empleados de una empresa saben más sobre su actividad que la propia empresa, o como cuando un vendedor de coches usados sabe más sobre éste que el futuro comprador. Un caso especialmente relevante de asimetría de la información es el relativo a la calidad de los productos que ofrecen los vendedores en el mercado, un caso muy estudiado en el mundo empresarial. En todos éstos casos, y en el mundo empresarial en general, es comúnmente aceptado el aserto "la información es poder".

La relación entre Economía y Dirección Estratégica es muy estrecha desde hace tiempo; la Estrategia empresarial es la relación lógica entre ambos conceptos, concretamente desde el lado cuantitativo. La principal misión de los "estrategas" de una empresa no es otra que la definición y planteamiento de metas, así como el desarrollo de planes para competir en los mercados. Todo esto viene a determinar que la estrategia debe materializarse a través de un proceso sistemático en el que la información tiene un papel central. La política competitiva en la empresa se apoya en la utilidad consistente en la posibilidad de que las empresas encuentren una situación en la industria para defenderse de fuerzas contrarias, o puedan influír a su favor. El análisis y conocimiento de 
éstas fuerzas es el que determina el "factor DAFO" de la empresa en cuestión. Pero éste conocimiento sólo se puede alcanzar logrando un conocimiento en profundidad de la competencia, algo que sólo se lograría por medio de cinco herramientas-procesos:

- evaluación de las estrategias actuales

- metas y objetivos futuros

- asunciones acerca de su posición y de la industria

- capacidades

- perfil del competidor

Otro concepto especialmente importante en el campo de la estrategia empresarial es la Ventaja Competitiva. La Ventaja Competitiva puede ser de dos tipos: de diferenciación, y de costes. El coste de la propia información es una variable muy a tener en cuenta; adquirir, almacenar y procesar conocimiento son actividades que implican costes, y los nuevos sistemas tecnológicos pueden reducir esos costes, a la par que mejorar la calidad de la información. Es entonces cuando la información se convierte en un elemento estratégico. Una Ventaja Competitiva sostenida en el tiempo de manera constante se deriva de los recursos y las capacidades empresariales que son valiosos y únicos en cada empresa. Es indudable pues, los beneficios que se obtienen de las inversiones en Tecnologías de la Información y Comunicación (TIC's) en una empresa. En algunos importantes foros se ha llegado a asegurar que los TIC's son otros recursos estratégicos más de una empresa, junto a los tradicionales como las patentes ó los derechos de propiedad intelectual, los copyrights, las bases de datos o los secretos comerciales, todos los cuales proporcionan capacidades, conocimiento e información, además de recursos propios.

El conocimiento está en un nivel diferente al de la información. Pero eso no quiere decir que las diferencias de conocimiento entre las distintas empresas sean el resultado de defectos del mercado, sino más bien de las distintas visiones que los empresarios tienen de su propia empresa y de la estrategia que aplican. El conocimiento no depende ni mucho menos de las máquinas, de los ordenadores, sino de los profesionales; la actual economía mundial es en muchos aspectos una Sociedad del Conocimiento, liderada por los "trabajadores del conocimiento", tal como los denominó en 2004 el experto P. Drucker. Ya mucho antes, en 1974, el premio Nobel e insigne economista austríaco, padre doctrinal del actual neoliberalismo económico, adversario ideológico de Keynes, e inspirador del también premio Nobel Milton Friedman, Friedrich Hayek, anunció la enorme importancia del conocimiento en la economía.

Efectivamente, la adquisición de conocimiento y su uso provechoso en forma de información es ya un factor determinante en la estrategia y en el éxito de una empresa. Se ha llegado a estudiar el porqué de que unas empresas estén más introducidas en el mercado que otras, partiendo de un análisis del conocimiento y uso por cada empresa. Un término, denominado "Marketing Orientation", estipula que las personas de una empresa deben ser conscientes 
de su papel como actores centrales del desarrollo de su propia empresa, a través tanto de sus capacidades como de su conocimiento. El resultado de todo es que a día de hoy la Información ya es considerada un producto, y no un bien o un servicio; un producto se puede comercializar, almacenar, distribuír, etc ... Eso es la información, y de ahí su poder en el mundo de la empresa.

Ya se avanzó hace treinta años (cuando nació Internet en 1981 y sólo tenía 1000 usuarios en todo el mundo, todos ellos grandes empresas) que la información y la tecnología revolucionarían la economía, afectándola en todos sus niveles, sin que ninguna empresa pudiera cerrarse las puertas ante las posibilidades que la información y la tecnología le ofrecerían. La realidad, treinta años después, ha dejado corta aquella predicción.

\section{La Competitive Intelligence y sus Conexiones con la Inteligencia Estatal-Militar}

Comencé éste trabajo haciendo una analogía entre la Guerra Fría y el mundo de la empresa, en lo relativo a la Inteligencia. $Y$ es que utilizar la información ya fue un tema central mucho antes de comenzar a aplicarse al ámbito empresarial y económico. Las primeras alusiones a la Inteligencia se remontan a las tres principales obras clásicas sobre Estrategia política y militar: "El arte de la guerra" de Sun Tzu, "El príncipe" de Maquiavelo, y "De la guerra", de Von Clausewitz. Se trata de obras de importancia capital en las que se detallan y recomiendan acciones concretas sobre Inteligencia y Contrainteligencia. Se centran en aspectos que actualmente continúan muy vigentes, tanto en el ámbito estatal-militar, como cada vez más, en el empresarial.

La primera institución moderna conocida en el mundo creada en exclusiva para desempeñar tareas de Inteligencia fue la Oficina de la División de Inteligencia Naval y del Ejército de los Estados Unidos, creada en 1880, y que inició su actividad dedicándose a la recopilación de información de carácter militar y de seguridad nacional en un país que había salido recientemente de una guerra civil y donde podían haber disidentes o incluso traidores. Actualmente en EE.UU hay cuarenta agencias de inteligencia, centradas cada una en un aspecto y un sector concreto de la Inteligencia, divididas entre las familias de la seguridad nacional, la seguridad militar y los servicios secretos, por lo que se habla de la "comunidad de inteligencia estadounidense". Destacan por su enorme tamaño y presupuesto la Central Intelligent Agency (CIA), la National Security Agency (NSA), la Federal Emergency Meeting Agency (FEMA), y la Defense Intelligent Agency (DIA), además del FBI, que tiene una misión mucho más policial y de lucha contra el crimen circunscribiendo sus actividades, a diferencia de las anteriores, al interior de EE.UU. Fuera de EE.UU, destacan el británico MI-6 (creado en la Iạ Guerra Mundial), el ruso FSB (antes KGB), el Mossad israelí, y curiosamente el servicio de inteligencia del Vaticano, centrado en actividades para-religiosas pero de una gran eficiencia. En España el servicio de inteligencia es el Centro Nacional de Inteligencia (CNI), denominado así desde 2002 y continuador de los anteriores CESID y SECED, servicios de inteligencia marcadamente militares. Los procesos usados por todas éstas 
agencias, y otras por todo el mundo, consistentes en la planificación, dirección, recopilación, procesamiento, análisis y formalización de la información, han sido todo un referente para la Competitive Intelligent. Muchas de sus prácticas han sido adaptadas al ámbito empresarial, si bien ciertas actividades propias de las agencias de inteligencia estatales se ciñen al campo del espionaje, completamente fuera de la Competitive Intelligent.

\section{Las Referencias Especializadas en el Campo de la Competitive Intelligent}

Las fuentes referenciales de las que se nutre la Competitive Intelligent aumentan constantemente en forma de revistas especializadas y referencias bibliográficas. Igualmente, la investigación en lo relativo a ésta nueva disciplina no deja de aumentar, y éste modesto trabajo es otra muestra.

Las revistas científicas más importantes en el mundo dentro del campo de la Competitive Intelligent son "Competitive Intelligent Journal", "Marketing Intelligent \& Planning", y "Journal of Competitive Intelligent \& Management". La mayor atención a la Competitive Intelligent se le prestó a finales de los 1990's, por lo que se puede hablar de un "boom" en esa época, pasando desde los años 1999 y 2000 a ser considerada ya no como una novedad, y sí una disciplina. Todo esto está redundando en la consideración de la Competitive Intelligent como disciplina propia dentro del área de la Administración de Empresas. Pero eso no significa que la Competitive Intelligent no sea el resultado de varias perspectivas, pues a día de hoy la inteligencia en las empresas es una actividad más integral, orientándose no sólo hacia el mercado de clientes y competidores, sino también hacia atrás en la cadena de valor de las empresas. La Competitive Intelligente es por ello todo un proceso, proceso que por supuesto no implica espionaje ni violar las leyes éticas y estatales.

Baste sólo con resumir las distintas denominaciones que se han referido para la Competitive Intelligent hasta la fecha:

- Inteligencia Tecnológica

- Inteligencia Económica

- Inteligencia Comercial

- Inteligencia Empresarial

Otra clasificación es la que divide a la Competitive Intelligent en tres niveles:

- Competitor Intelligent

- Market Intelligent

- Technological Intelligent 


\section{Competitive Intelligent: Una Visión Global}

Es evidente que el auge de la Competitive Intelligent en las tres últimas décadas se basa por un lado en las innovaciones tecnológicas, y por otro en una mayor disponibilidad de información, una enorme proliferación en bases de datos comerciales por todo el mundo. Además de esto, otra variable ha facilitado su desarrollo: la Globalización, que ha supuesto una explosión en la competitividad empresarial a escala planetaria. Ya no se trata sólo, como sucedía hasta finales de los 1980's, de empresas multinacionales estadounidenses, europeas y japonesas compitiendo por el mercado global: ahora están además empresas coreanas, chinas, de Taiwán y otros "dragones asiáticos", sin olvidar algunas de economías latinoamericanas emergentes como Brasil ó Méjico. En éste clima económico mundial enormemente competitivo, la Competitive Intelligent está creciendo cada vez más en relevancia y utilidad.

Ya se trate de conocer una industria, un mercado, un producto novedoso o un competidor, la información fiable es básica para una gran empresa. La manipulación electrónica de datos y su almacenamiento en microchips están siendo fenómenos con un impacto similar al de la invención de la imprenta en la Europa del Siglo XV. Así mismo, el acceso a un mundo de información prácticamente ilimitado le da a las grandes empresas actuales la posibilidad de entenderse a sí mismas y a sus mercados de forma eficiente.

La Competitive Intelligent, como ya se ha dicho en éste trabajo, no consiste en espiar, sino en reunir sistemática y legalmente toda la información posible para su análisis detenido que posteriormente, ya "digerida" esa información, será usada para obtener un mayor entendimiento de la estructura, la cultura, el comportamiento, las capacidades y las debilidades de la empresa competidora. En ocasiones se precisa una gran cantidad de información para analizar al competidor, pero en otras basta con una pequeña y precisa información; en el avanzado campo de la robótica, por ejemplo, ceñido a unas pocas empresas en todo el mundo, bastaría para controlar a una empresa competidora con descubrir qué pequeña pero importante innovación tecnológica se está preparando en una planta de una factoría de esa empresa competidora, e incluso solamente bastaría con averiguar qué maquinaria están usando en dicha planta. En ocasiones se han hecho similitudes entre la Competitive Intelligent y la $\mathrm{CIA}$, en cuanto a descubrir secretos del adversario; además, se sabe que la CIA tiene en Langley (Virginia) una avanzadísimo y gran equipo formado por cientos de personas con el sólo propósito de analizar y procesar información, descartando aquella información no-útil (la mayoría) y concentrándose en la útil. Sin embargo, las similitudes desaparecen a partir de ahí. La CIA en infinidad de ocasiones ha traspasado los márgenes de la ley, y lo mismo se puede decir del mundo del espionaje, mientras que la Competitive Intelligent nunca lo hace. Definitivamente, la Competitive Intelligent no tiene nada que ver con el espionaje.

Los objetivos fundamentales de la Competitive Intelligent son, básica y sintéticamente, cuatro: 
- identificar amenazas competitivas

- eliminar o disminuír sorpresas

- mejorar la ventaja competitiva disminuyendo el tiempo de reacción

- encontrar nuevas oportunidades

El alcance de la Competitive Intelligent es tan amplio que puede usar información relacionada con prácticamente cualquier producto o actividad, así como de tendencias o temas industriales, e incluso tendencias geopolíticas (como por ejemplo la concentración del comercio mundial en las dos orillas del Pacífico). También puede ser capaz de detectar amenazas futuras, como la gestación de un próximo competidor que está a punto de nacer en un sector concreto. Un ejemplo cercano en el tiempo se produjo en la década de los 1970's, cuando nace el mercado mundial de los ordenadores personales. IBM, el dominador mundial de sistemas informáticos hasta entonces, se encontró de repente con dos inesperados y hasta entonces inexistentes competidores: Hewlett-Packard (surgido en 1972 en Syllicon Valley y que comenzó a fabricar computadoras a comienzos de los 1970's) y Apple (nacido en 1978 cuando dos jóvenes estudiantes, Steve Jobs y Steve Wozniak, desarrollaron el primer y rudimentario PC en un garaje de San Francisco). IBM no podía saber que éstos dos con el tiempo feroces competidores iban a disputarle su cetro, y sin embargo ésta lucha, y la incorporación de otros gigantes como Dell o NCR, transformó para siempre el negocio de la hasta entonces hegemónica IBM.

El ejemplo anterior es una buena muestra de cómo aplicando una adecuada Competitive Intelligent, o bien una ausencia de ésta, puede determinar la marcha de un negocio durante décadas. Apple surgió casi de repente, pero un buen análisis competitivo pudo haberlo advertido a tiempo, tomando decisiones estratégicas como por ejemplo hubiera sido, por parte de IBM, incorporar a esos dos jóvenes a su equipo neutralizando así la futura amenaza que finalmente supusieron con la creación de Apple. A veces sucede que es muy difícil detectar esas amenazas a tiempo, y en ocasiones las amenazas que suponen futuros competidores son simple e insensatamente despreciadas: así sucedió en la década de los 1960's en el sector automovilístico estadounidense, que dominaba el mercado estadounidense y mundial desde Detroit, con marcas como Ford, Chrysler, Mustang, Docge y otras varias. Desde esa ciudad, capital de la industria del automóvil de Estados Unidos se llegó a decir que los estadounidenses sólo comprarían coches estadounidenses, por su calidad y diseño. Veinte años después, ya en los 1980 's, los coches japoneses inundaban el mercado estadounidense ante la impotencia de Detroit, comprados en masa por la clase media estadounidense por su calidad, y los coches europeos se convertían en los más cotizados en EE.UU, aunque menos vendidos que los japoneses, sobretodo marcas alemanas como BMW o Mercedes. Desde los 1990's se incorporarían los coches coreanos, y en la actualidad en torno a un $40 \%$ del mercado estadounidense está en manos de marcas extranjeras, algo inconcebible hasta la década de los 1960's y en buena parte debido a la poca seriedad con que los fabricantes de Detroit se tomaron la competencia que se avecinaba. 
Éste ejemplo de los automóviles, válido para muchos otros sectores, sirve para ilustrar el hecho de que no todas las empresas, aunque sean grandes multinacionales, llegan a entender verdaderamente la naturaleza y la dinámica de su propio sector y de sus consumidores potenciales. Incluso no todas las empresas o divisiones de éstas entienden siempre sus intereses; cuando falta tal visión, que sólo puede ser proporcionada por un buen entendimiento, ninguna aplicación de Competitive Intelligent podrá evitar el desastre. En ausencia de ese entendimiento, las amenazas son difíciles de identificar. Esto es exactamente la Competitive Intelligent, y en esto consiste: detectar amenazas y maneras de lograr la información necesaria una vez que la amenaza es avistada.

Los "ejecutores" de la Competitive Intelligent, es decir, las personas que llevan a cabo tareas y procedimientos que hacen posible la Competitive Intelligent, son de diversos tipos: van desde "ratas de biblioteca", analistas de centros de información, expertos analistas financieros, planificadores, comerciales, e incluso personal directivo interesado en darle un rumbo concreto a la Competitive Intelligent en curso. Algunas grandes empresas, y también firmas privadas de consultoría que se dedican a ofrecer sus servicios en Competitive Intelligent e investigación industrial a grandes empresas, incorporan entre éste personal dedicado a éstas labores a exanalistas militares y de inteligencia retirados, así como a especialistas en información y del mundo docente. Se trata de personal muy cualificado y acostumbrado a llevar a cabo éstas tareas.

A nivel asociativo, la Competitive Intelligent ha proliferado desde la década de los 1980's; a parte de la asociación más relevante, la SCIP, existen otras, como la Information Industry Association, el Planning Forum, la American Marketing Association, e incluso la Special Library Association, todas ellas relacionadas de diversas formas a las necesidades de información. Esto a hecho que la Competitive Intelligent haya crecido en los últimos treinta años y haya desarrollado sus propias escuelas de pensamiento, sus propios "gurús" (autores de palabras clave), y sus propias tendencias. Sin embargo, en todas estas tendencias está instalado el nexo común de no transgredir la frontera de lo ético ni de la legalidad.

Dentro de éste análisis interno de la Competitive Intelligent como disciplina, a nivel doctrinal se han desarrollado tres escuelas. La primera sostiene que la interpretación y el análisis son fundamentales en la Competitive Intelligent, ya sean análisis numéricos o comparativos, ya que determinar las herramientas de análisis más adecuadas para comunicar los índices de una empresa respecto a sus posibles competidores no es tarea sencilla. La segunda escuela cree que la recolección y "caza" de la información es la verdadera actividad fundamental. El único escollo es lograr la técnica adecuada para discriminar diariamente la información no-útil, centrándose en la útil, y cómo poder mantenerse actualizado con la enorme proliferación de archivos y bases de datos así como fuentes de información diversas, y al mismo tiempo cómo poder actualizarse con los procedimientos para conseguir información que no está publicada y visible. La tercera escuela opina que la recolección de información fiable y su análisis son importantes a partes iguales, y la una no puede prevalecer sobre la 
otra, y si una de ellas está debilitada, la otra se resentirá, fallando así todo el proceso de Competitive Intelligent.

Es un hecho que la información sin procesar o el traspaso de datos tienen una utilidad muy escasa, y se trata de material no apto para ser entregado al cliente final, ya que deben ser integrados y analizados en un documento bien organizado y fácil de interpretar y que incluya gráficos, comparativas, tablas y demás ayudas. Otra cuestión importante es el hecho de que si se desconoce la clase de información que se va a encontrar durante el proceso de "caza y recolección", es difícil llegar a formular las preguntas adecuadas. Así, tener una base de datos consistente en la datación de, por ejemplo, el movimiento comercial de mercancías de una empresa por todo el mundo, es un instrumento que permite a un analista rastrear el movimiento de mercancías de una empresa a otra extranjera. Sólo conociendo éste recurso, un gerente puede fácilmente preguntarse qué productos han sido comercializados por una empresa determinada en un determinado espacio de tiempo, llegando así a conclusiones importantes.

La dirección de una empresa puede no llegar a formular las preguntas correctas sobre el escenario competitivo sin entender los tipos de información que dan forma a ese escenario. Es ahí donde entra la Competitive Intelligent, usando para ello estudios y campañas para informar a la dirección de la empresa, dirección que ya habría dado el primer paso para implementar tareas de Competitive Intelligent, pero antes entendiendo qué tipos de información se podrían encontrar, algo básico para que esa Competitive Intelligent tuviera éxito. Una vez iniciado el estudio de Competitive Intelligent, el uso de información pública para elaborar esos estudios oscilaría entre un $80-90 \%$ de la información total usada. A la hora de encontrar la información, es importante saber que ésta hay que buscarla, pero al final está donde se encuentra, en los lugares a veces más insospechados. También es importante saber que en la mayoría de las veces, y como sucede con casi todo, una investigación de Competitive Intelligent pasa por varias etapas que suelen ser secuencialmente así: primera etapa ascendente y optimista, segunda etapa en caída tras comprobar las dificultades, y tercera y definitiva etapa en la que la investigación va tomando forma, hasta alcanzar la meta deseada.

Como sucede con cualquier tipo de investigación (periodística, policial), en la Competitive Intelligent no es aconsejable usar una única fuente de información, ya que puede tratarse de una fuente poco fiable o rigurosa. Es aconsejable verificar a la primera fuente de información con otra $u$ otras fuentes. Toda información que no pueda verificarse a través de al menos una segunda fuente debe ser tratada como un simple rumor. Otra cuestión importante a la hora de investigar y recopilar información es el hecho de que las empresas dejan, consciente o inconscientemente, un rastro al hacer negocios, rastro que puede ser por supuesto rastreado. Es un hecho que "por cada acción hay una reacción". Si una empresa que fabrica neumáticos está intentando producir un nuevo tipo de neumático que involucra nuevos y revolucionarios materiales químicos, está obligada a comunicarlo a determinadas instancias estatales, lo cual dejará un rastro y con ello pistas a sus competidores. Si esa misma 
empresa quiere expansionarse en, por ejemplo, el mercado latinoamericano, sus representantes deben viajar al país latinoamericano elegido y solicitar los permisos y licencias oportunos, lo cual supone documentos que llegado el caso pueden ser consultados. Se trata de formas de investigar perfectamente legales. Al final, una buena Competitive Intelligent supone entender el mundo empresarial suficientemente como para hallar los rastros (documentos, solicitudes) que generan las decisiones y los actos de una empresa.

Y es que la Competitive Intelligent usa básicamente información pública, y es en éste punto donde debemos definir claramente qué es algo público, y en concreto definir qué es publicar algo: se trata de "hacer algo conocido, público, poniéndolo de manifiesto ". Así, algo es público cuando "es accesible y compartido por cualquiera". Son definiciones simples, no rebuscadas, pero enormemente clarificadoras. En el ámbito empresarial, el que una empresa tenga datos o documentos públicos no significa necesariamente que desee diseminarlos a los cuatro vientos; simplemente son rastros que va dejando en sus actividades, rastros reveladores sobre sus negocios. Hay otros datos sin embargo que las empresas desean dejar en silencio, que no trasciendan, datos tales como planes estratégicos a varios años, desarrollos de mercados, nuevos productos o expansiones comerciales. Se trata de datos ocultos, no públicos, y en éste terreno la Competitive Intelligent no actúa; actuaría el espionaje industrial. $Y$ es que cuando una empresa tiene una tecnología novedosa 0 mejorada respecto a la que ya existe sabe que puede tener un producto que la puede convertir en la fuerza dominante de un sector; pero un competidor que se maneja rápidamente a través de una mejor comprensión del mercado y una habilidad para compensar el no haber tenido esa innovación tecnológica, puede sin embargo tener éxito al preparar el camino y ganarle la partida a la primera empresa.

De nuevo conviene insistir en que una buena investigación de Competitive Intelligent puede usar información pública en un 80-90\% de la información total a usar. El resto puede calcularse o deducirse. Un método muy a tener en cuenta es poder detectar adecuadamente cuales son los canales de información verdaderamente productivos y cuales los que proporcionan información poco útil, para centrarse en los primeros. Otra distinción importante es no confundir la información pública con la información publicada; mucha información pública pero no publicada son por ejemplo permisos, licencias, solicitudes, documentos oficiales, etc ... de una empresa competidora que se dispone a instalar una nueva planta en una provincia o municipio, dando claras pistas de sus intenciones. $Y$ es que los formularios oficiales suelen contener información muy fiable. Incluso existen bases de datos comerciales especializadas en formularios oficiales. Pero información oficial-documental aparte, en todo lo relativo a datos sobre empresas, los directorios de empresas y corporaciones son excelentes fuentes de información sobre éstas intenciones comerciales basadas en número de empleados o cifras de esa nueva planta o inversión. Algunos importantes directorios que comercializan éste tipo de información son "Dun's Million Dollar Directory", el "Standard \& Poor's Register of Corporations", la "Encyclopedia of Associations", y el "Directory of Corporate Affiliations". 
Otro tipo de información corporativa sobre grandes empresas puede consultarse con total libertad en informaciones que diariamente se emiten de forma abierta y pública: el índice Dow-Jones de la bolsa de Nueva York (y el índice de cualquier bolsa del mundo), los diarios financieros Wall Street Journal o Financial Times y otros, así como datos publicados semanalmente en toda clase de diarios, periódicos y revistas de economía. Incluso un periódico local de una pequeña población donde va a instalarse próximamente una industria de la competencia es una excelente fuente de información para averiguar intenciones de la competencia.

En Estados Unidos, gracias a importantes estudios de Competitive Intelligent llevados a cabo desde finales de los 1980's y comienzos de los 1990's, se puso en evidencia que en general, el nivel de calidad de los productos estadounidenses estaba lejos del nivel de calidad de los europeos y los japoneses. La penetración masiva a mediados de los 1980's de los automóviles japoneses y alemanes en EE.UU fue sólo una muestra de lo que estaba pasando. Otros sectores como los electrodomésticos y la alta tecnología también daban muestras similares ya entonces de debilidad frente a la competencia de fuera. La conclusión era clara: en un mundo que ya a finales de los 1980's comenzaba a globalizarse, Estados Unidos y sus corporaciones debían incrementar su gasto en $\mathrm{I}+\mathrm{D}+\mathrm{I}$ si quería poder competir con sus equivalentes europeos y japoneses (y hoy en día con coreanos, chinos y otros). El mercado doméstico estadounidense, tradicionalmente monopolizado por los productos "made in USA" estaba cambiando, se estaba abriendo a los productos extranjeros, que penetraban gracias a su mayor calidad proporcionada por una mayor inversión en investigación, una tendencia ya consolidada veinticinco años después. Baste con dar un dato: desde hace veinte años, el Masachussets Institute of Technology recibe millones de dólares de las multinacionales japonesas, que por supuesto aprovechan a ésta institución para invertir y recuperar su inversión en forma de innovación, sirviéndose así de las instituciones y el talento de su competidor, Estados Unidos, patrocinándolas.

\section{Conclusiones y Generalidades}

Para la instauración de una unidad de inteligencia en una empresa haría falta, en primer lugar y de forma lógica, la voluntad de instaurarla, pero acto seguido los pasos técnicos serían: incubación, concepción, implementación, estructuración y evaluación. Una vez puesta en marcha la unidad, sus fases de funcionamiento ya en forma de Competitive Intelligent son: planificación y enfoque, recolección, análisis, comunicación, proceso y estructura. Simultáneamente se deben llevar a cabo tareas consistentes en identificar a los proveedores, indentificar a posibles fuentes de información, recopilar, evaluar la validez, comprobar la utilidad de la información recogida, integrar, interpretar, realizar la planificación estratégica o táctica, elaborar conclusiones y recomendaciones, diseminar y presentar los resultados, y como colofón final, el feedback. 
Un aspecto a destacar es la evolución de la Competitive Intelligent en los últimos treinta años, cuando a partir de 1980 comienza a emerger como una disciplina cada vez más autónoma. Antes de 1980, la labor se ceñía básicamente a la toma de datos de la competencia, pero en la década de los 1980 's se avanza hacia el análisis industrial y de la competencia. En los 1990's se instaura de lleno la Competitive Intelligent, y se prevé que el futuro consista en una Competitive Intelligent como una parte esencial de una empresa, un departamento más dentro de una empresa. En ésta evolución también se observa el grado de "seriedad" con el que se trata a la Competitive Intelligent, pasando de básicamente la informalidad hace treinta años, hacia la creación de unidades formales y a la combinación de ambas hoy día. También en la orientación, pasando de una puramente táctica a una estratégica, más amplia. En cuanto al análisis, se ha pasado de uno pequeño y limitado a uno más extenso, cualitativa y cuantitativamente. El proceso de toma de decisiones se ha visto afectado al principio de una forma muy limitada, para pasar a un grado débil, luego fuerte, y actualmente directo. Los modos de obtener datos eran básicamente bibliotecas y marketing a comienzos de los 1980's, planificación avanzada la década, marketing avanzado ya en los 1990's, y una mezcla optimizada de planificación y marketing a día de hoy. Finalmente, los problemas se iniciaron en su desarrollo de habilidades para lograr la información en 1980, la elaboración de simulaciones de Competitive Intelligent avanzada la década, ya en los 1990's crece la demanda y la oferta de Competitive Intellgent y se emplea la tecnología, y en la actualidad se tiende a administrar los procedimientos de inteligencia, sobretodo en grandes empresas.

Un aspecto especialmente reseñable es el relativo a la confusión de la Competitive Intelligent con el espionaje. $Y$ es que con demasiada frecuencia es confundida la Competitive Intelligent con el espionaje industrial. La gran diferencia estriba en que mientras todos sabemos en qué consiste el espionaje, ya sea industrial o gubernamental, la Competitive Intelligent supone la recabación de información de forma ética y legal, ya que se trata de información disponible de una u otra forma para el público; se trata sólo de recabarla de forma eficiente y eligiendo la que es útil de la inútil. En el espionaje se emplean métodos ilegales y "oscuros". La Competitive Intelligent no implica ninguno de éstos elementos.

En definitiva, para hacer un estudio aunque sea básicamente introductorio, como pretendo con éste trabajo, sobre Competitive Intelligent, se deben revisar todas las teorías existentes sobre el tema, además de apoyarse en la Teoría Económica y de la Información, en las Teorías de la Estrategia, en la Ventaja Competitiva, en los Recursos y Capacidades, en el Conocimiento, y en el Marketing. 